Introduction Neisseria gonorrhoeae resistance to extended-spectrum cephalosporins as well as treatment failures with ESCs has been increasingly reported in many countries globally. These increasing trends together with the limitation of drugs of choice lead gonorrhoea to become a global health concern. Herein, we aimed to reveal more understanding on the physiological response of gonococci to ESCs using proteomic approach.

Methods N. gonorrhoeae reference strain was grown with or without a subinhibitory dose of ESCs. Protein expression was determined by 2-dimensional gel electrophoresis in conjunction with MALDI-TOF/TOF MS analysis.

Results In total, 14 and 13 proteins were significantly altered expression following exposure to ceftriaxone and cefixime, respectively. Most of expressed proteins shared a similar expression pattern in response to ceftriaxone and cefixime reflecting similarities in antibiotic mechanisms. ESC antibiotics triggered proteins in a variety of functions, such as membrane protein, transport system, energy metabolism, and stress response, which would help gonococci to survive under drug stress. Interestingly, the subinhibitory dose of ESCs also triggered the expression of gonococcal virulence factors (e.g. azurin and peptidyl-prolyl isomerase), which might be an adaptation mechanism of gonococci in ESC stress and also affect the outcome of gonococcal infection.

Conclusion The present work might provide new insights into physiological adaptive networks of gonococci to antimicrobial agents and more understanding toward the mechanism of action, which subsequently may benefit for the further drug discovery of new antimicrobials to combat with resistant gonococci.

\section{P05.09 PHENOTYPIC, GENETIC AND GENOMIC CHARACTERISATION OF THE 2015 WHO NEISSERIA GONORRHOEAE REFERENCE STRAINS FOR QUALITY ASSURANCE OF LABORATORY INVESTIGATIONS GLOBALLY}

${ }^{1} \mathrm{M}$ Unemo*, ${ }^{1} \mathrm{D}$ Golparian, ${ }^{2} Y$ Grad, ${ }^{3} \mathrm{~A}$ Limnios, ${ }^{4} \mathrm{~T}$ Wi, ${ }^{3} \mathrm{M}$ Lahra, ${ }^{5} \mathrm{~S}$ Harris. ${ }^{1}$ WHO Collaborating Centre for Gonorrhoea and Other Sexually Transmitted Infections, National Reference Laboratory for Pathogenic Neisseria, Department of Laboratory Medicine, Clinical Microbiology, Faculty of Medicine and Health, Örebro University, Örebro, Sweden; ${ }^{2}$ Department of Immunology and Infectious Diseases, Harvard TH Chan School of Public Health, Boston, MA, USA; ${ }^{3}$ WHO Collaborating Centre for STD, Microbiology Department, The Prince of Wales Hospital, Randwick, Sydney, Australia; ${ }^{4}$ Department of Reproductive Health and Research, World Health Organization (WHO), Geneva, Switzerland; ${ }^{5}$ Pathogen Genomics, The Wellcome Trust Sanger Institute, Wellcome Trust Genome Campus, Cambridgeshire, UK

\subsection{6/sextrans-2015-052270.295}

Introduction Gonorrhoea and antimicrobial resistance (AMR) in Neisseria gonorrhoeae are major public health concerns globally. Resistance to all antimicrobials available for treatment of gonorrhoea has now been reported in N. gonorrhoeae. Enhanced quality assured gonococcal AMR surveillance is crucial worldwide and the WHO Global Gonococcal Antimicrobial Surveillance Programme (GASP) was revitalised in 2009. To obtain reliable and comparable AMR data internationally, appropriate and wellcharacterised $N$. gonorrhoeae reference strains are essential for quality assurance. The phenotypic and genetic characteristics of the 2008 WHO N. gonorrhoeae reference strains $(n=8)$ were previously published. Here, we describe the phenotypic, genetic, and genomic characteristics of the 2015 WHO N. gonorrhoeae reference strains.
Methods In the 2015 WHO N. gonorrhoeae reference strain panel $(\mathrm{n}=14)$, six additional strains have been selected to include representation of high-level cephalosporin and azithromycin resistance and porA mutant strain. These strains were phenotypically characterised by antibiogram, serovar, and prolyliminopeptidase (PIP) screening; and genetically in regards of resistance plasmid types, polymorphisms in divergent genetic resistance-mediating loci $(\mathrm{n}=14)$, porB sequencing, $N$. gonorrhoeae multiantigen sequence typing (NG-MAST), and multilocus sequence typing (MLST). Fully characterised finished reference genomes for all the 2015 WHO N. gonorrhoeae reference strains were produced using PacBio and Illumina sequencing technologies.

Results The 2015 WHO reference strains represent all available main phenotypes of resistance and susceptibility to antimicrobials previously and currently used for treatment of gonorrhoea, as well as several considered for future use. All corresponding resistance genotypes and molecular epidemiological types were also elucidated. Finally, references genomes of each strain were obtained and characterised in detail.

Conclusion The 2015 WHO N. gonorrhoeae reference strains are intended for internal and external quality assurance in all types of laboratory investigations, i.e. particularly in GASP, but also for phenotypic (e.g. culture) and molecular diagnostics, species determination, genetic AMR detection, molecular epidemiology, and genome sequencing as well as other novel molecular technologies.

Disclosure of interest statement This work was funded by the Örebro County Council Research Committee and the Foundation for Medical Research at Örebro University Hospital, Sweden, the WHO, and The Wellcome Trust Sanger Institute, Wellcome Trust Genome Campus, Cambridgeshire, United Kingdom.

\section{P05.10 ANTIMICROBIAL RESISTANCE OF NEISSERIA GONORRHOEA IN GERMANY, RESULTS FROM THE GONOCOCCAL RESISTANCE NETWORK (GORENET)}

${ }^{1} \mathrm{~S}$ Dudareva-Vizule, ${ }^{2} \mathrm{~S}$ Buder, ${ }^{1} \mathrm{~K}$ Jansen* ${ }^{*},{ }^{1} \mathrm{~A}$ Loenenbach, ${ }^{3,4} \mathrm{~S}$ Nikisins, ${ }^{1} \mathrm{~A}$ Sailer, ${ }^{2} \mathrm{E}$ Guhl, ${ }^{2}$ PK Kohl, 'V Bremer. 'Department for Infectious Disease Epidemiology, Robert Koch Institute, Berlin, Germany; ${ }^{2}$ German Reference Laboratory for Gonococci, Department of Dermatology and Venerology, Vivantes Hospital Berlin, Germany; ${ }^{3}$ Department of Infectious Diseases, Robert Koch Institute, Berlin, Germany; ${ }^{4}$ European Public Health Microbiology Training (EUPHEM) Programme, European Centre for Disease Prevention and Control (ECDC), Stockholm, Sweden

\subsection{6/sextrans-2015-052270.296}

Introduction Neisseria gonorrhoeae (NG)-infections are not reportable in Germany and only limited data on NG-epidemiology and antimicrobial resistance (AMR) are available. With GORENET we monitor the NG-AMR and patterns of resistance testing in Germany in order to guide treatment algorithms and targeted prevention strategies.

Methods We recruited laboratories based on geographic distribution and number of NG-isolates. From April 2014 prospective data on all performed NG-AMR-tests together with patientrelated information were collected. Laboratories send a part of the isolates to the national reference laboratory (NRL) for culturing and AMR-testing towards ceftriaxone, cefixime, azithromycin, ciprofloxacin, and penicillin by using E-Test, as well as beta-lactamase. Results are interpreted according to European Committee on Antimicrobial Susceptibility Testing 4.0. 
We described the reported samples by sex and age and characterised isolates tested in NRL by resistance patterns. We calculated proportions and medians, together with interquartile range (IQR), where appropriate.

Results Between April and December 2014 we received information on 651 isolates tested in 19 laboratories. Altogether, 90.3\% isolates were from men and $8.5 \%$ from women. Median age of tested men was 36 (IQR 29-49) and women 28 (IQR 22-41) years.

NRL received 502 isolates, 342 were vital and 253 were tested for AMR. From those 0 were resistant towards ceftriaxone, $1.6 \%$ towards cefixime, $11.1 \%$ towards azithromycin, $73.1 \%$ towards ciprofloxacin, and $30.4 \%$ towards penicillin. Further $37.9 \%$ and $50.6 \%$ isolates were intermediate susceptible to Azithromycin and to Penicillin. From 205 isolates tested for beta-lactamase, $25.9 \%$ were positive.

Conclusion In Germany isolates tested for NG-AMR were mostly from men. We assume that a substantial proportion of these isolates could be from men having sex with men. NGAMR to ceftriaxone and cefixime remains low, while resistance and intermediate susceptibility to azithromycin, ciprofloxacin and penicillin is substantial. Monitoring of NG-AMR should be highly prioritised.

Disclosure of interest statement The GORENET Project is funded by German Federal Ministry of Health. No conflicts of interest.

\section{P05.11 ANTIMICROBIAL SUSCEPTIBILITY OF NEISSERIA GONORRHOEAE STRAINS FROM MALE URETHRITIS IN JAPAN: SURVEILLANCE AT 2012-2013}

\begin{abstract}
${ }^{1,2} \mathrm{R}$ Hamasuna*${ }^{2},{ }^{2} \mathrm{M}$ Yasuda, ${ }^{2} \mathrm{~K}$ Ishikawa, ${ }^{2} \mathrm{~S}$ Uehara, ${ }^{2} \mathrm{H}$ Hayami, ${ }^{2} \mathrm{~S}$ Takahashi, ${ }^{2} \mathrm{~T}$ Matsumoto, ${ }^{2} \mathrm{~S}$ Yamamoto, ${ }^{3} \mathrm{H}$ Hanaki, ${ }^{2} \mathrm{~J}$ Kadota. ${ }^{1}$ Department of Urology, University of Occupational and Environmental Health, Japan; ${ }^{2}$ The Members of Urogenital SubCommittee and the Surveillance Committee of Japanese Society of Chemotherapy (JSC), Japanese Association for Infectious Diseases (JAID) and Japanese Society for Clinical Microbiology (JSCM), Japan; ${ }^{3}$ Research Center for Anti-Infectious Drugs, Kitasato University, Japan
\end{abstract}

\subsection{6/sextrans-2015-052270.297}

Introduction Neisseria gonorrhoeae is one of the most important pathogens causing sexually transmitted infection. Resistant $N$. gonorrhoeae trains against several antimicrobials are increasing worldwide. In this study, the trends of antimicrobial susceptibilities among N. gonorrhoeae strains isolated from male patients with urethritis were investigated as the second Japanese national surveillance, which was conducted by a Japanese surveillance committee.

Methods The targets were male patients older than 16 years with urethral discharge and symptoms of urethritis. Urethral discharge was collected from patients at 26 medical facilities from March 2012 to January 2013 and the antimicrobial susceptibility of strains were tested.

Results From 151 patients (median age 32 years), 103 N. gonorrhoeae strains were tested for susceptibility to 20 antimicrobial agents. None was susceptible to penicillin G (MIC: $\leq 0.06 \mu \mathrm{g}$ / $\mathrm{mL}$ ). The prevalence of penicillinase producing $N$. gonorrhoeae or chromosomally mediated resistant N. gonorrhoeae was $1.9 \%$ or $20.8 \%$, respectively. The susceptible rate for fluoroquinolone such as ciprofloxacin or levofloxacin was $20.3 \%$. The susceptible rate for cefixime was $89.3 \%$, that decreased from $98.8 \%$ at 2009. No ceftriaxone-resistant strains founded. The prevalence of less susceptible strain to ceftriaxone (MIC: $0.125 \mu \mathrm{g} / \mathrm{mL}$ ) was 10.7\%. No azithromycin high-resistant strain found and 5 strains (4.9\%) had MIC $\geq 0.5 \mu \mathrm{g} / \mathrm{mL}$. Among all strains, 83 strains $(80.6 \%)$ were resistant to more than 2 kinds of antimicrobials and 42 strains $(40.1 \%)$ was included to the criteria as multi-drug resistant $N$. gonorrhoeae.

Conclusion The antimicrobial susceptibility of N. gonorrhoeae was not different between 2009 and 2012-2013, in Japan.

Disclosure of interest statement Nothing to declare.

\section{P05.12 ANALYSIS OF BACTERIAL FLORA OF THE URINE SPECIMENS FROM MALE PATIENTS WITH URETHRITIS BY THE CLONE LIBRARY METHOD BASED ON THE $16 \mathrm{~S}$ RRNA GENE}

${ }^{1} \mathrm{R}$ Hamasuna*, ${ }^{2} \mathrm{CL}$ You, ${ }^{2} \mathrm{~K}$ Fukuda, ${ }^{1} \mathrm{~N}$ Fujimoto, ${ }^{3} \mathrm{~T}$ Hachisuga, ${ }^{1,4} \mathrm{~T}$ Matsumoto, ${ }^{2} \mathrm{H}$ Taniguchi. ${ }^{1}$ Department of Urology, University of Occupational and Environmental Health, Japan; ${ }^{2}$ Department of Microbiology, University of Occupational and Environmental Health, Japan; ${ }^{3}$ Department of Obstetrics and Gynecology, University of Occupational and Environmental Health, Japan; ${ }^{4}$ Medical Officer, Kitakyuushu City

\subsection{6/sextrans-2015-052270.298}

Introduction Chlamydia trachomatis, Mycoplasma genitalium or Trichomonas vaginalis are the pathogens for non-gonococcal urethritis, but pathogenicity of other microorganisms for the male urethral has not be confirmed. In this study, the bacterial flora of urine specimens from male patients with urethritis was analysed by the clone library method based on the 16S rRNA gene. Methods The first voided urine specimens of male patients with urethritis were collected. The detection of Neisseria gonorrheae, C.trachomatis were examined by transcriptional mediated amplification (TMA) method and M. genitalium, Ureaplasma urealyticum, Ureaplasma parvum and Mycoplasma hominis were examined by real-time PCR method. The urine specimens were strained by Ethidium bromide and the number of bacterial cells were counted. DNA was extracted from the urine and amplification of the 16S rRNA gene via PCR (universal primers) and determination of the nucleotide sequences of 96 colonies were performed. The homology search was performed with a basic local aligment search tool (BLAST) using in-house software system and phylotypes of bacteria in each specimens were analysed. Results Urine specimens were collected from 58 patients and the divided to 4 group; gonogoccal urethritis (n:9), chlamydial urethritis (n:12), non-chlamydial non gonococcal urethritis detecting with Ureaplasmas or Mycoplasmas (UM+NCNGU, n;15) and non-chlamydial non gonococcal urethritis detecting without Ureaplasmas or Mycoplasmas (UM-NCNGU, n:22). Cell numbers were higher in $\mathrm{UM}+\mathrm{NCNGU}$ than in UM-NCNGU. Among specimens, 2427 clones in 38 bacterial flora could be analysed and 91 bacterial phylotypes were detected together with N.gonorrhoeae, C.trachomatis, Mycoplasmas or Ureaplasmas positive specimens among chlamydial urethritis or $\mathrm{UM}+\mathrm{NCNGU}$ groups. Gardnerella vaginalis was detected with Mycoplasma/Ureaplasma in Chlamydial urethritis ot UM+NCNGU.

Conclusion The clone library method by using 16S rRNA gene is the new technology for analysis the bacterial flora of urine with urethritis. The relationship between G. vaginalis and Ureaplasma/Mycoplasma was found by this analysis.

Disclosure of interest statement This study was supported by Japan Society for Promotion Science (JSPS) KAKENHI Grant Number 25293337. 